\title{
DOCUMENT DETECTION SUMMARY OF RESULTS
}

\author{
Donna Harman \\ National Institute of Standards and Technology \\ Gaithersburg, MD. 20899
}

\section{INTRODUCTION}

This section presents a summary of the TIPSTER results, including some comparative system performance and some conclusions about the success of the detection half of the TIPSTER phase I project. For more details on the individual experiments, please see the system overviews.

Four contractors were involved in the document detection half of TIPSTER. Two of the contractors worked in English only (Syracuse University and HNC Inc.), one contractor worked in Japanese only (TRW Systems Development Division), and one contractor worked in both languages (University of Massachusetts at Amherst). The four contractors had extremely varied approaches to the detection task. TRW transformed an operational English retrieval system (based on pattern matching using a fast hardware approach), into a Japanese version of the same operation, with a special interface designed to facilitate work in Japanese. The University of Massachusetts approach involved taking a relatively small experimental system using a probabilistic inference net methodology, scaling it up to handle the very large amounts of text and long topics in TIPSTER, and modifying the algorithms to handle Japanese. Both Syracuse University and HNC Inc. built completely new systems to handle the English collection. In the case of Syracuse University, their system is based heavily on a natural language approach to retrieval, with many of the techniques traditionally used in document understanding applied to the retrieval task. HNC Inc. took a totally different approach, applying statistical techniques based on robust mathematical models (including the use of neural networks).

There were three evaluations of the contractors' work; one at 12 months, one at 18 months, and the final one at 24 months. In each case, the contractors working in English have made multiple experimental runs using the test collection, and tumed in the top list of documents found. These results were first used to create the sample pool for assessment, and then were scored against the correct answers based on results from all runs (including TREC-1 runs for the 18-month evaluation and TREC-2 runs for the 24-month evaluation). Standard tables using recall/precision and recall/fallout measures were distributed and compared. The evaluation of the Japanese work took place only at the 24 -month period.

\section{12-MONTH EVALUATION}

The work done for the 12-month evaluation was mainly a scaling effort. Not all data was available so only parial results were completed. In particular, the University of Massachusetts tumed in 7 runs using the adhoc topics, with experiments trying different parts of the topic to automatically create the query, and also adding phrases. Additionally they tried some manually edited queries. HNC Inc. turned in 4 runs using the adhoc topics, with experiments also using different parts of the topic to automatically generate queries. Additionally they tried various types of "bootstrapping" methodologies to generate context vectors. Syracuse University turned in no runs, but had completed the extensive design work as proposed in their timeline. The University of Massachusetts also did 4 runs on the routing topics, but the lack of good training data made this very difficult. In general the results for the systems was good, with the University of Massachusetts outperforming HNC Inc. on the adhoc runs, but it was felt by all that this evaluation represented a very "baseline" effort. For these reasons, no graphs of these results will be presented.

\section{18-MONTH EVALUATION}

By the 18-month mark, the systems had finished much more extensive sets of experiments. The University of Massachusetts continued to investigate the effects of using different parts of the topic for the adhoc runs, but this time trying different combinations using the inference net methodology. Figure 1 shows three INQRY runs for the adhoc topics done for the 18-month evaluation. The plot for INQRYV represents results from queries created automatically using most of the fields of the topics. The INQRYJ results are from the same queries, but including phrases and concept operators. The INQRYQ results 
show the effects of manually editing the INQRYJ queries, with those modifications restricted to eliminating words and phrases, adding additional words and phrases from the narrative field, and inserting paragraph-level operators around words and phrases. As can be seen, the use of phrases in addition to single terms helped somewhat, but the results from manual modification of the queries were the best adhoc runs.

Figure 1 also shows the two HNC adhoc results for the 18-month evaluation period. These runs represent final results from many sets of bootstrapping runs in which HNC evolved techniques for completely automatically creating context vectors for the documents. The plot marked $\mathrm{HNC2aV}$ represents the results using these context vectors for retrieval and using automatically built queries from the concepts field of the topic. The HNC2aM results adds a rough Boolean filter to the process, requiring that three terms in the concepts field match terms in the documents before the context vectors are used for ranking. This Boolean filter provided considerable improvements.

Figure 2 shows the routing results for the 18-month evaluation. The three plots for the INQRY system represent a baseline method (INQRYA, same as INQRYJ adhoc approach), and two modifications to this method. The INQRYP results show the effect of manually modifying the query, similar to the method used in producing the INQRYQ adhoc results. The plot for INQRYR shows the first results from probabilistic query expansion and reweighting performed automatically using relevance feedback techniques on the training data. Both methods improve results, with the automatic feedback method results approaching the manual-modification method, especially at the high recall area of the curve.

The HNC routing results shown on figure 2 represent the use of two different types of neural networks. The plot marked $\mathrm{HNCrtl}$ is the baseline result, created by using the adhoc methods similar to those used in run HNC2aV. The HNCr12 results represent using neural network techniques to learn improved stem weights for the context vectors based on the training data. The HNCrt3 results come from using the training data to determine what type of routing query to use, i.e. an automated adhoc query (similar to HNC2aM), a manual query, or a query using the neural network techniques (HNCrt2). Clearly the neural network learning techniques significantly improve performance, with the per topic "customization" performance (HNCrt3) working best.

In terms of system comparison, the University of Massachusetts runs were consistently better than the HNC runs for the adhoc topics, whereas for the routing topics both groups were similar. The results were a major im- provement over their baseline (12-month) results for both groups.

At the 18-month evaluation period, Syracuse University had the first stage of their system in operation and turned in results for the first time. The results for adhoc and routing are shown in figures 3 and 4 . Since the results are for only a subset of the data used by the other contractors, they cannot be directly compared. Additionally since the results are only for the first stage of retrieval, which emphasizes high recall, they should not be viewed as the final results from the system.

Figure 3 shows four Syracuse runs on the adhoc topics. The documents used are the subset of the collection having the Wall Street Journal only. The first three plots, DRsfc1, DRpna1, and DRtsal, represent three operations in the DR-LINK system. The first operation does a rough filtering operation on the data, only retrieving documents with suitable subject field codes. The next two operations locate proper nouns and look at document structure. There is a considerable improvement in performance between the first two operations. The fourth run (DRfull) used a manual version of the second stage to produce final results. These results are for only half the topics, so cannot be strictly compared to the first three runs, but they do indicate the potential improvements to precision that can be expected from the second stage.

Figure 4 shows the same operations, and generally the same improvements, for the routing topics. In this case the subset of documents used was the AP newswire documents. The same three operations discussed above are plotted here. There was no second stage trial for the routing topics. These two graphs represent the baseline of the Syracuse system.

\section{24-MONTH EVALUATION}

For the 24-month evaluation, all groups turned in many runs. The runs were much more elaborate, with many different types of parameters being tried. The University of Massachusetts tried 7 experiments with the adhoc topics, using complex methods of combining the topic fields, proximity restrictions, noun phrases, and paragraph operators. Additionally an automatically-built thesaurus was tried. They also did 15 runs with the routing topics, trying various experiments combining relevance feedback, query combinations, proximity operators and special phrase additions. HNC Inc. did 4 adhoc runs using various types of learned context vectors. Additionally they tried a simulated feedback query construction run. For routing they did 5 runs, trying multiple experiments in different combinations of adhoc and neural net approaches. Syracuse University tumed in 10 runs for their 


\section{TIPSTER 18 Month Adhoc}
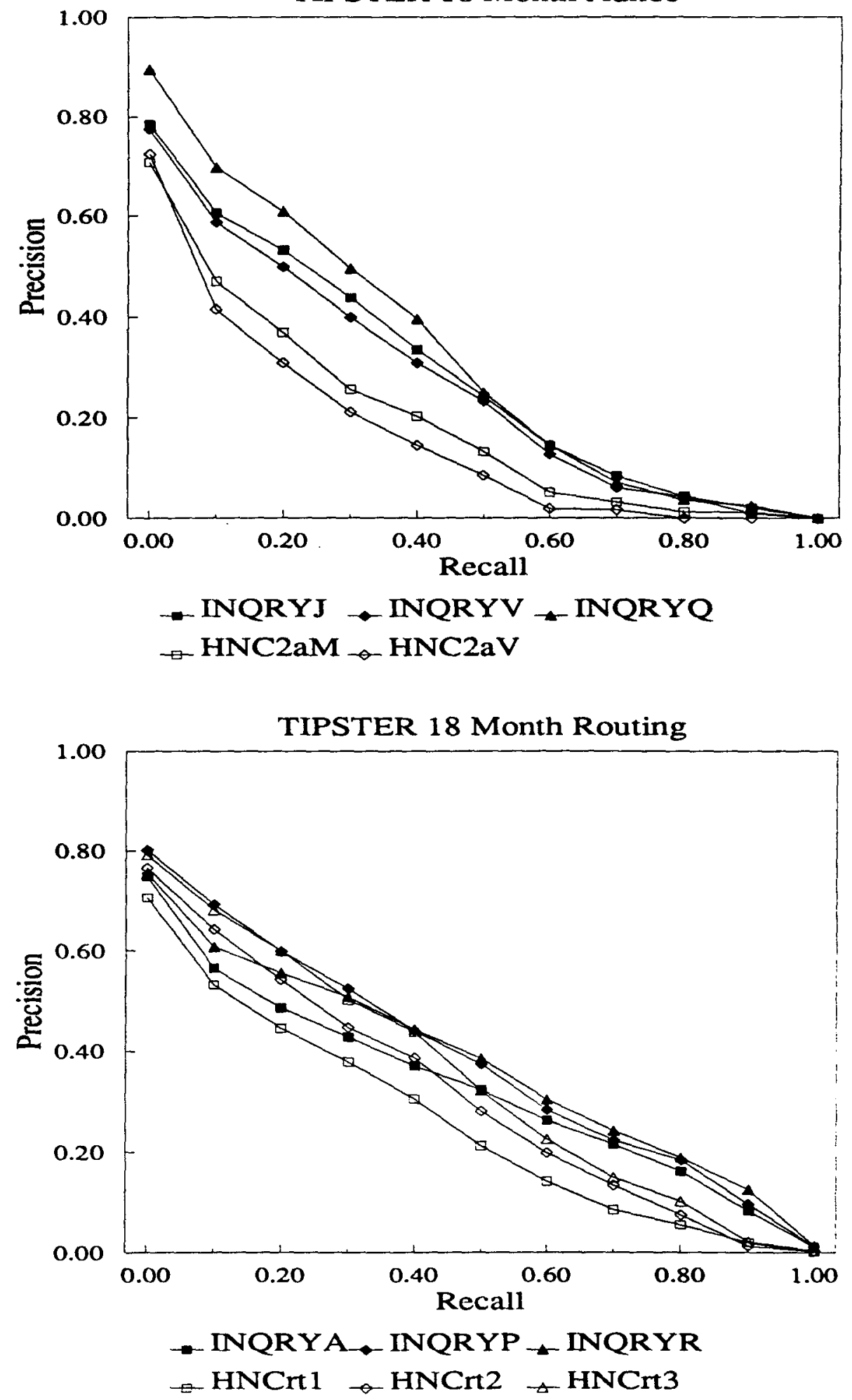

Figures 1 and 2: Adhoc and routing performance at the 18-month evaluation period (using full collection) 

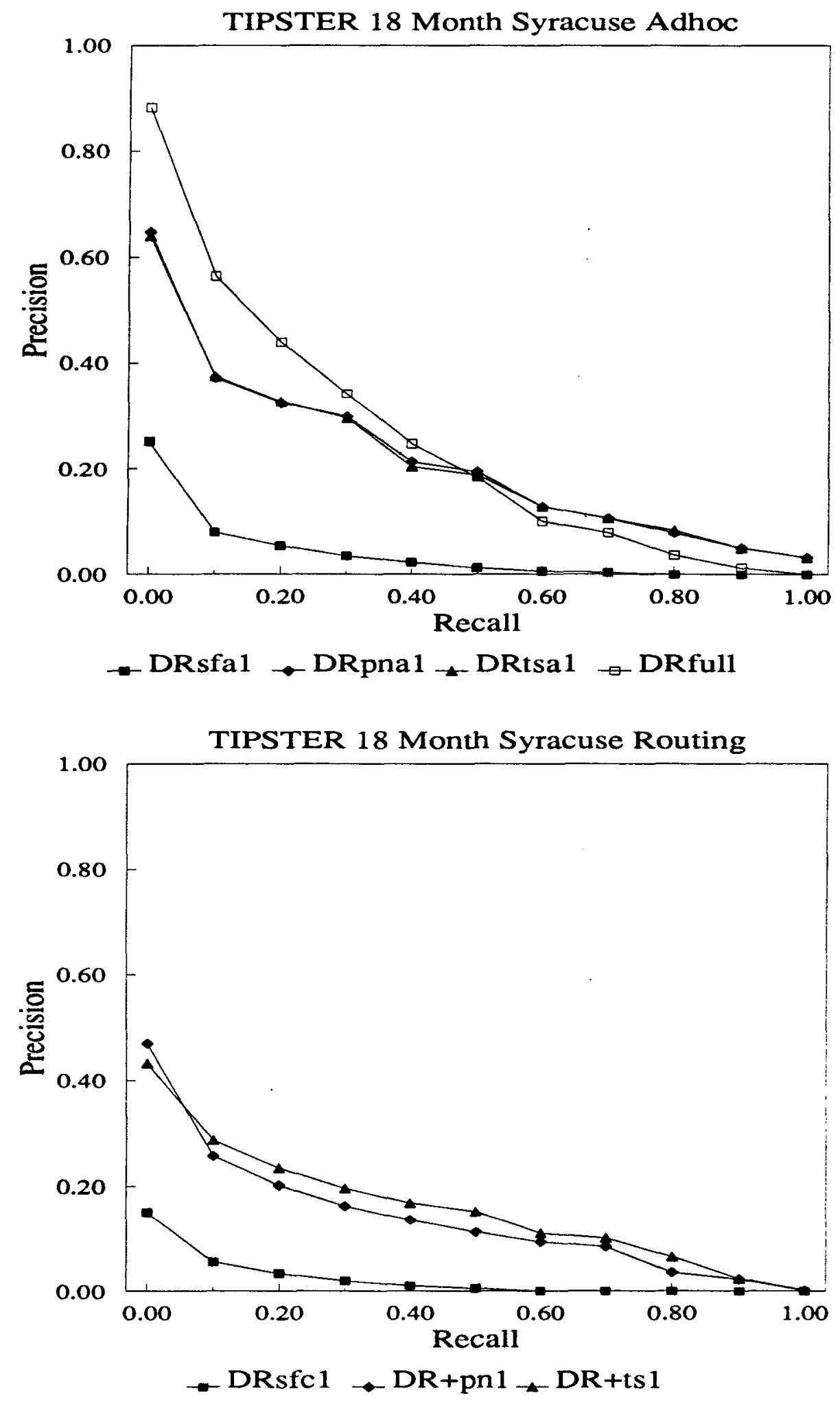

Figures 3 and 4: Adhoc and routing performance at the 18-month evaluation period (using WJS or AP only) 
"upstream processing module" ( 3 adhoc and 7 routing), trying various types of ranking formulas. Additionally they did 13 runs using the full retrieval system (4 adhoc and 9 routing). Full descriptions of these runs are given in the system overviews.

Figures 5 through 12 show the results from the 24-month evaluation. Figures 5 and 6 show some of the adhoc results for the full collection, and figures 7 and 8 show some of the routing results. The results from Syracuse University on a smaller subset of the document collection are shown in figures 9 through 12 .

Figure 5 shows the recall/precision curves for the adhoc topics. The three INQRY runs include their baseline method (INQ009), which is same as the baseline method INQRYJ developed at the 18-month evaluation period. The first modification (INQ012) uses the inference net to "combine" weights from the documents and weights from the best-matching paragraphs in the document. The second modification (INQ015) shows the new term expansion method using an automatically-built thesaurus. Both modifications show some improvements over the baseline method.

The three HNC runs shown on figure 5 include a baseline (HNCad1) that is similar to their best 18-month adhoc approach (HNC2aM), but that uses a required match of 4 terms rather than 3 . The HNCad3 results show the effects of using a larger context vector of 512 terms rather than only 280 terms for the baseline results. This causes a slight improvement. The HNCad2 results are using some manual relevance feedback.

The University of Massachusetts results are better than the HNC results, but there were improvements in both systems over the 18 -month evaluation. Figure 6 shows the recall/fallout curves for the best runs of these two systems. Both plots show the same differences in performance, but it can be seen on the recall/fallout curve that both systems are retrieving at a very high accuracy. At a recall of about 60 percent (i.e. about 60 percent of the relevant documents have been retrieved) the precision of the INQRY results is about 30 percent. The fallout, however, is about 0.0004 , meaning that most non-relevant documents are being properly screened out. This corresponds to a probability of false alarm rate of 0.0004 at this point, in ROC terminology.

Figure 7 shows the routing results for both groups. The run marked INQ026 is the baseline run of the INQRY system and uses the same methodology as the adhoc INQ009 run. The other two runs add some type of relevance feedback using the training documents. The plot marked INQ023 uses both relevance feedback and proximity operators to add 30 terms and 30 pairs of terms from the relevant documents to the query. The most complex run,
INQ030, constructed the queries similarly to run INQ023, but additionally weighted the documents using a combination method similar to adhoc run INQ012. These runs represented the best results from many different experiments, and the relevance feedback gives significant improvement over the baseline runs.

The HNC routing results also represent the best of many experiments. The results for HNCrts show the neural network learning using stem weighting, similar to $\mathrm{HNCrt}$ at the 18-month evaluation. The second two sets of results represent data fusion techiques, with $\mathrm{HNCrt} l$ being fusion of four types of retrievals, using the same combinations for all topics, and HNCrt2 using different combinations for different topics. The data fusion combinations both work well, but the per topic combination works the best, just as the less sophisticated version of this run worked best at the 18-month evaluation.

Again the University of Massachusetts results were better than the HNC results, but with major improvements in both systems over the 18-month evaluation. Figure 8 shows the recall/fallout curves for the best runs of both groups.

The Syracuse runs were on a subset of the full collection so are not directly comparable. However they also showed significant improvements over their 18-month baseline. Figure 9 shows three first-stage Syracuse runs, the results of trying different complex methods of combining the information (subject field code, text structure, and other information) that is detected in the first-stage modules. The results of this combination are passed to the second stage (figure 10). Note that due to processing errors there were documents lost between stages, and these official results are therefore inaccurate. Additionally only 19 topics (out of 50) are shown in figure 10. The improvements that could have been expected do not show because of these problems.

Figures 11 and 12 show the Syracuse routing runs. The first stage runs show not only the combinations from the adhoc, but also additional ways of integrating the data. Again there were processing errors with the second stage results, and therefore no improvement is shown using the second stage. 
TIPSTER 24 Month Adhoc
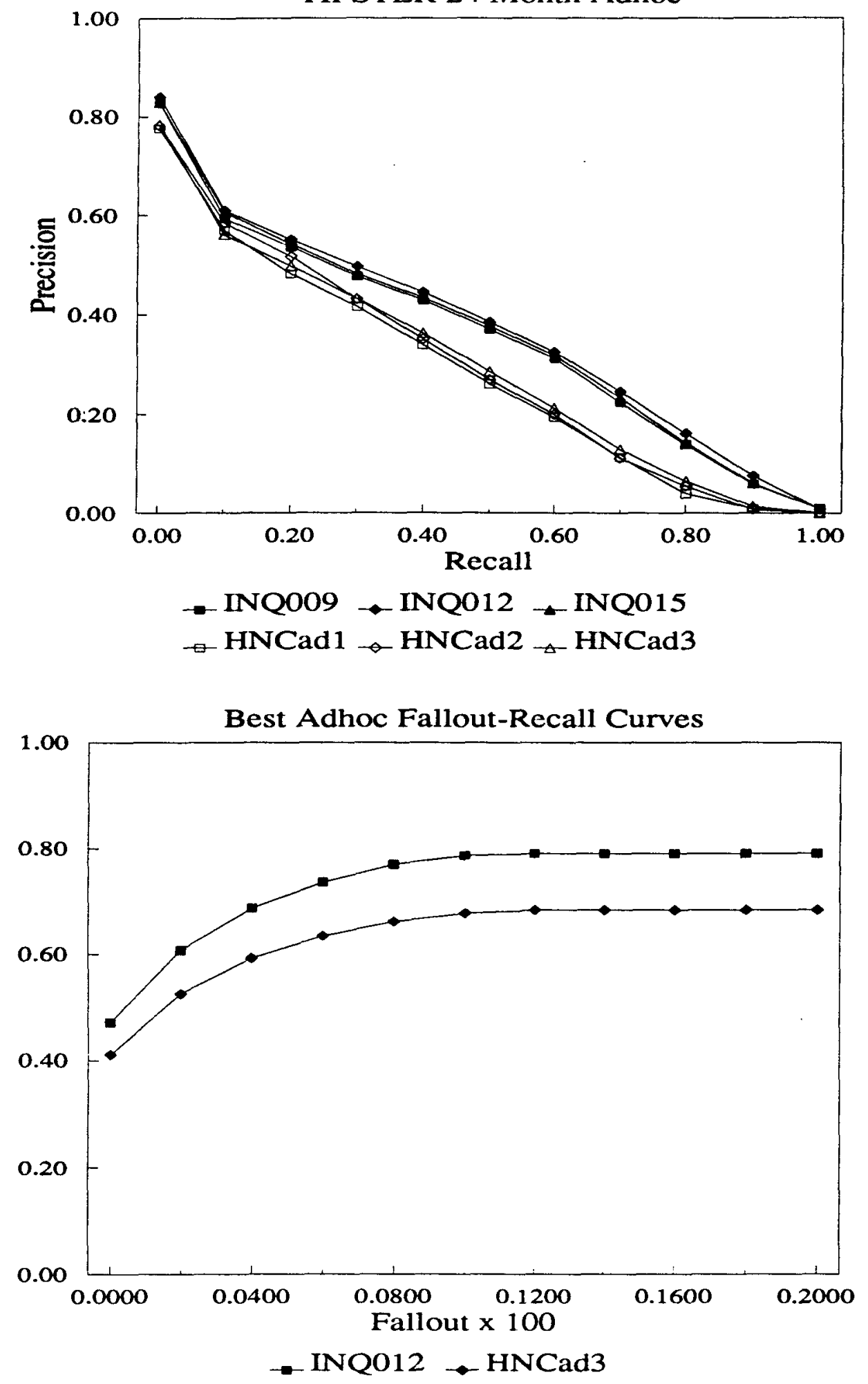

Figures 5 and 6: Adhoc performance at the 24-month evaluation period (using full collection) 
TIPSTER 24 Month Routing
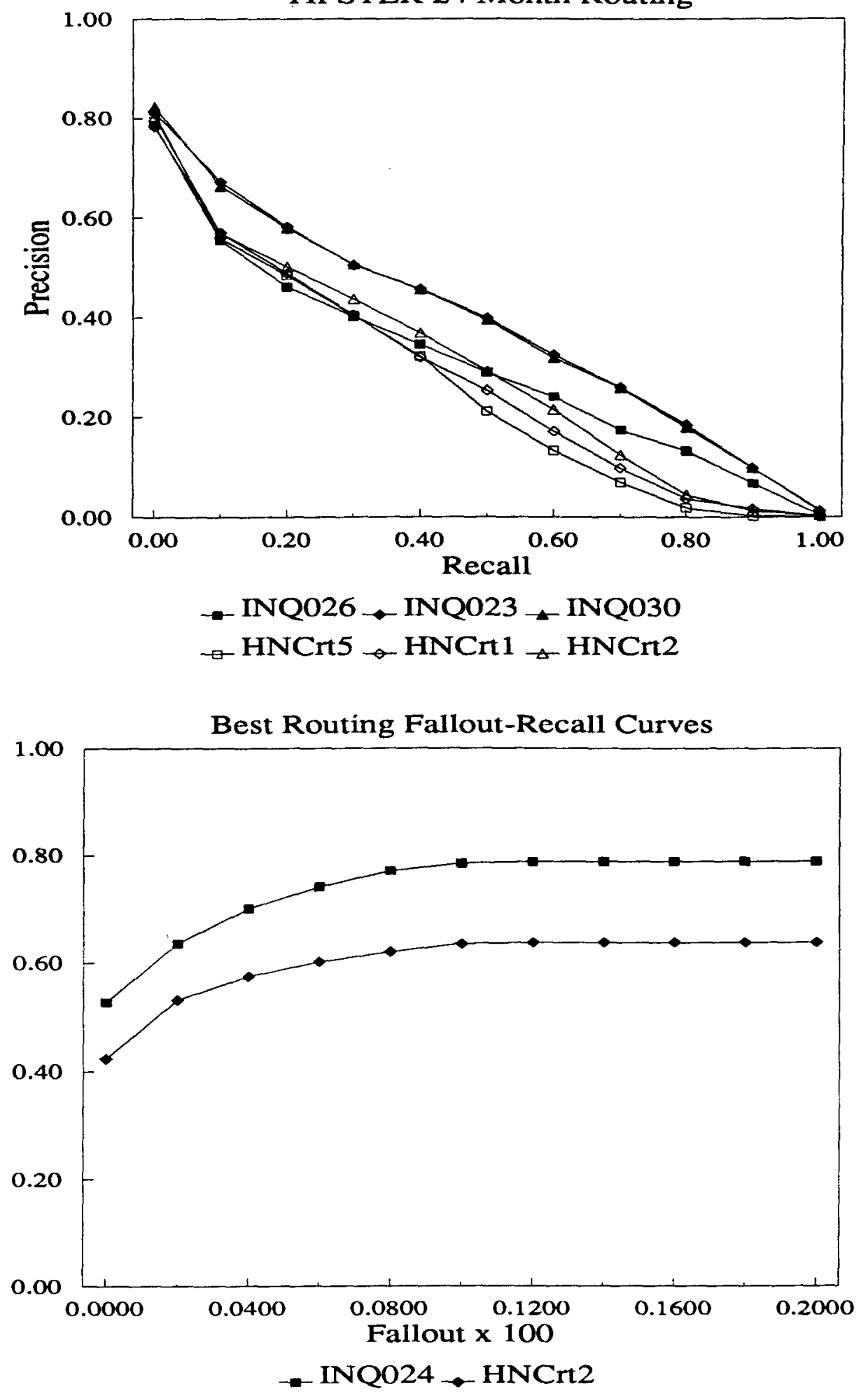

Figures 7 and 8: Routing performance at the 24-month evaluation period (using full collection) 

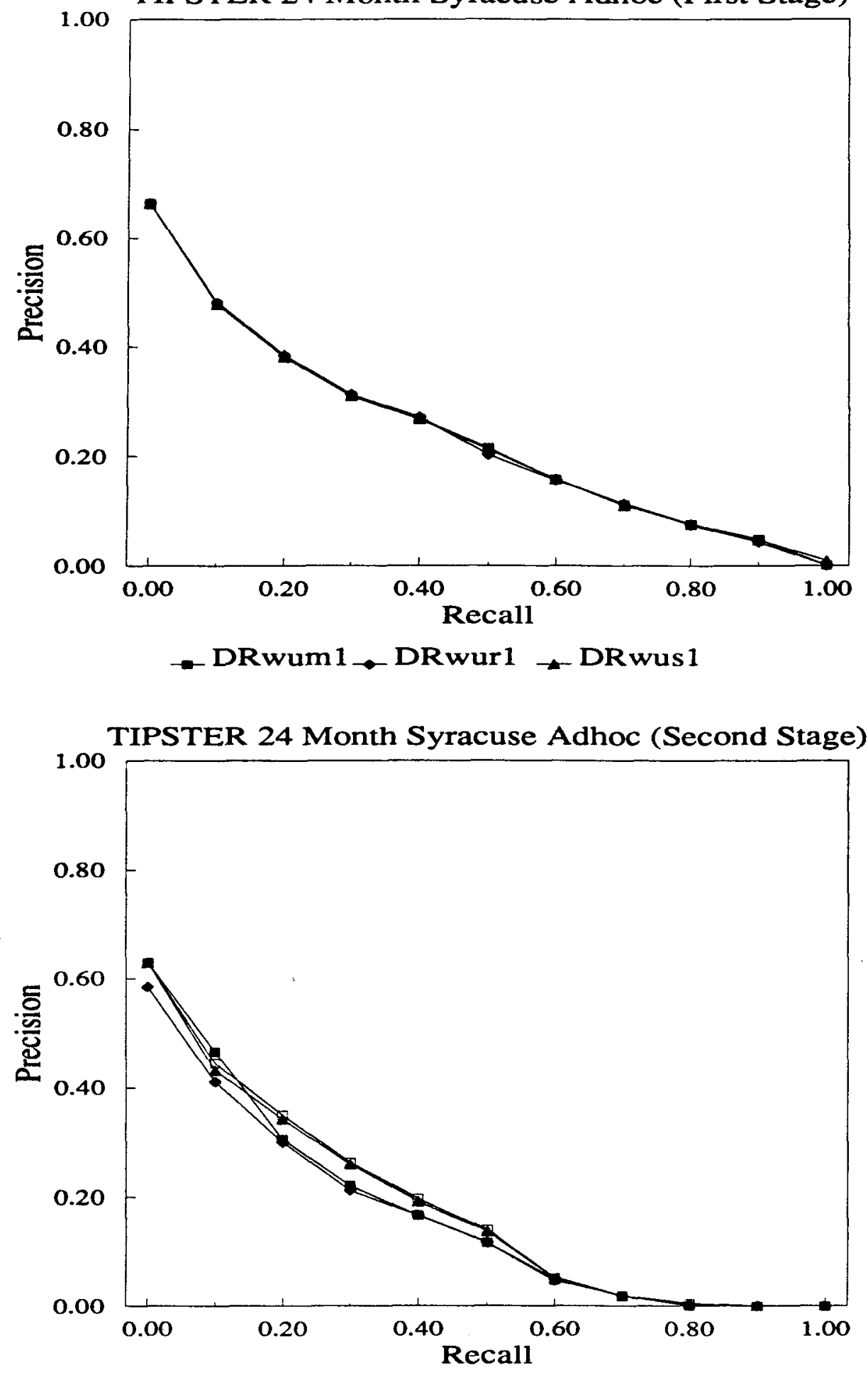

$-\mathrm{DR} 1 \mathrm{bw} 2 \multimap \mathrm{DR} 2 \mathrm{bw} 2 \nrightarrow \mathrm{DR} 3 \mathrm{bw} 2 \multimap \mathrm{DR} 4 \mathrm{bw} 2$

Figures 9 and 10: Adhoc performance at the 24-month evaluation period (using WJS only) 

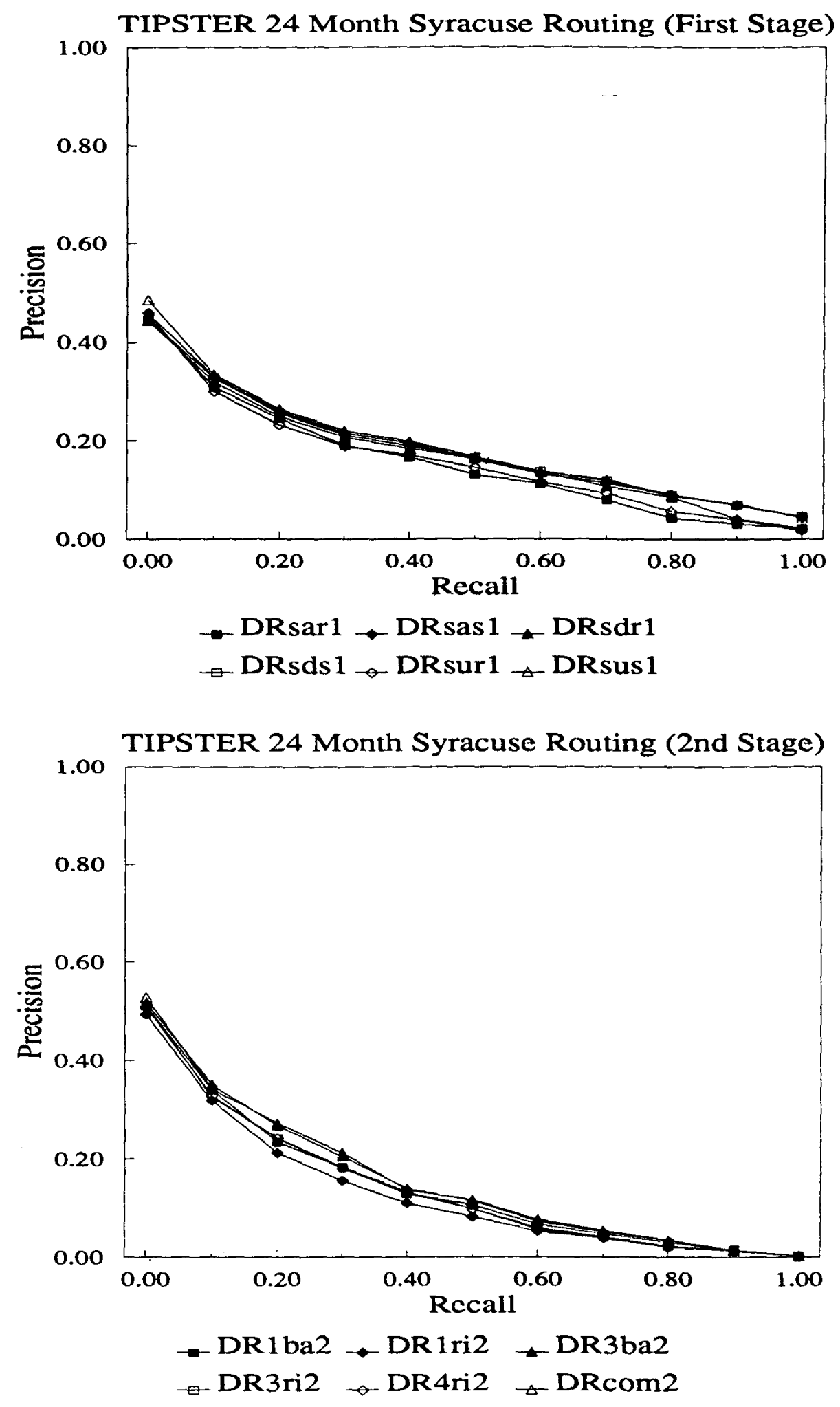

Figures 11 and 12: Routing performance at the 24-month evaluation period (using SJMN only) 


\section{COMPARISON WITH TREC RESULTS}

How do the TIPSTER results compare with the TREC-2 results? Two of the TIPSTER contractors submitted results for TREC-2 and these can be seen in Figures 13 and 14. These figures show the best TREC-2 adhoc and routing results for the full collection. More information about the various TREC- 2 runs can be found in the TREC-2 proceedings [1]. The results marked "INQ001" are the TIPSTER INQUERY system, using methods similar to their baseline TIPSTER INQ009 run. The "dortQ2", "Brkly3" and "crnlL2" are all based on the use of the Cornell SMART system, but with important variations. The "cmlL2" run is the basic SMART system, but using less than optimal term weightings (by mistake). The "dortQ2" results come from using the training data to find parameter weights for various query factors, whereas the "Brkly3" results come from performing statistical regression analysis to learn term weighting. The "CLARTA" system adds noun phrases found in an automatically-constructed thesaurus to improve the query terms taken from the topic. The plot marked "HNCad1" is the baseline adhoc run for the TIPSTER 24-month evaluation. The TIPSTER INQUERY system is one of the best performing systems for the TREC-2 adhoc topics.

The routing results from TREC-2 (shown in figure 14) exhibit more differences between the systems. Again three systems are based on the Cornell SMART system. The plot marked "cmiCl" is the actual SMART system, using the basic Rocchio relevance feedback algorithms, and adding many terms (up to 500) from the relevant training documents to the terms in the topic. The "dortP1" results come from using a probabilistically-based relevance feedback instead of the vector-space algorithm. These two systems have the best routing results. The "Brkly5" system uses statistical regression on the relevant training documents to learn new term weights. The "cityr2" results are based on a traditional probabilistic reweighting from the relevant documents, adding only a small number of new terms (10-25) to the topic. The "INQ003" results also use probabilistic reweighting and add 30 new terms to the topics. The "hnc2c" results are similar to the HNCrtl fusion results for the 24-month TIPSTER evaluation.

These plots mask important information as they are averages over the 50 adhoc or routing topics. Whereas often the averages show litule difference between systems, these systems are performing quite differently when viewed on a topic by topic basis. Table 1 shows the "top 8" TREC-2 systems for each adhoc topic. The various system tags illustrate that a wide variety of systems do well on these topics, and that often a system that does not do well on average may perform best for a given topic. This is an inherent performance characteristic of information retrieval systems, and emphasizes the importance of getting be- yond the averages in doing evaluation. Clearly systems that perform well on average reflect better overall methodologies, but often much can be learned by analyzing why a given system performs well or poorly on a given topic. This is where more work is needed with respect to analyzing the TIPSTER and TREC results.

Tables 2 and 3 show some preliminary analysis of two of the topics with respect to the TIPSTER contractors. Table 2 gives the ranks of the relevant documents retrieved either by the HNCrt1 nun or the INQ023 run. Clearly the HNC run is better for this topic, providing much higher ranks for most of the relevant documents. Note that five of the relevant documents were not retrieved by either system.

Table 3 shows a slightly different view of the same phenomena, but for topic 121 . There were a total of 55 relevant documents for this topic, with only 13 of them found by the TIPSTER systems. Table 3 lists those 13 documents, the rank at which they were retrieved, and the "tag" of the system retrieving them. Note that for this topic the INQUERY system is performing better than the HNC system. These tables illustrate the varying performance of different methods across the topics. A major challenge facing each group is to determine which strategies are successful for most topics, and which strategies are successful only for some topics (including how to identify in advance this topic subset).

\section{JAPANESE RESULTS}

By the 24-month evaluation, only 7 topics were ready for testing. Both TRW and the University of Massachusetts ran these topics successfully, and the results are discussed in their system evaluations. No comparison of the results is possible between the two systems because of the preliminary nature of having only 7 topics. However, the University of Massachusetts (who did both English and Japanese) reported that minimal effort was necessary for porting their English techniques to Japanese, especially given the availability of the JUMAN Japanese word segmentor. Additionally the new TRW Japanese interface was judged a major success by the beta site tests.

\section{CONCLUSIONS}

What are some of the conclusions that can be drawn from the many experiments performed in the TIPSTER and TREC evaluations, and equally important, what is the lasting value of this two-year project?

First, the statistical techniques (using non-Boolean methods without any formal query language) that were used on the smaller test collections DO scale up. The simplest example of this is the consistently high performance of the Comell SMART system in TREC. This very basic system 
relies on the vector-space model and on carefully crafted term weighting to produce their high results. A more complex example of the successful use of statistical techniques is the University of Massachusetts INQUERY system, which uses the more sophisticated inference network approach to achieve their high performance. This system has been very successful throughout the TIPSTER project, and has achieved this success using variations on their original system rather than having to completely revise their techniques.

Second, the results obtained by the best systems in TIPSTER and TREC are at a level of performance that is generally accepted to be superior to the best current Boolean retrieval system. More importantly, this performance is achieved from simple natural language input, allowing consistently superior retrieval performance without exhaustive training or experience. These systems are clearly ready to be tested in fully operational environments.

Third, the use of a large test collection has shown some unexpected results. Techniques that should have brought improvements have not done so. The use of phrases instead of single terms has not resulted in significant improvements; the use of proximity or paragraph-level retrieval has not shown especially good results; and the use of more complex NLP techniques have not worked well yet. Conversely, techniques that have not been successtul before such as using types of automatic thesaurii for topic expansion have had unexpected success. These unexpected results using a large test collection are reopening research on old discarded ideas and starting research in new areas. It is much too early to draw firm conclusions on any of these techniques. Often poor performance that is attributed to one problem may be the result of lack of balance in parameter adjustment, e.g., the lack of improvement from phrases may be caused by the difficulty in balancing the weights of these phrases and the weights of single terms.

What is the lasting value of the document detection half of the TIPSTER phase I project? The first contribution in my opinion has been the development of a large test collection and the wide acceptance of its use via the TREC conferences. The lack of a large test collection has been a major barrier in the field of information retrieval and its removal allows an expansion of research by many groups world-wide.

The second lasting value is the demonstration of the feasibility of using the non-Boolean, statistically-based retrieval systems both in the ARPA community and in the broader commercial sector. Not only have wellestablished small-scale research groups braved the scaling effort, but at least four new commercial products have used the TIPSTER/TREC program as launching pads.
The TIPSTER program has caused the establishment of two major new retrieval research groups; both Syracuse University and HNC Inc. have built systems during the TIPSTER project that are approaching the power of the best of the TIPSTER/TREC systems. Additionally many of the TREC systems are either new groups in the information retrieval research arena or are older groups expanding their small programs to tackle this major retrieval experiment.

The final lasting value of the TIPSTER project has been the joining of the NLP community and the information retrieval (IR) community in the project. This has led to the high expections for combining these disjoint technologies in phase II and has helped cement the important collaboration of two diverse groups of researchers.

These three lasting contributions are not only of value individually, but will lead to a resurgence of research in the information retrieval area. The combination of the large test collection, the growing demand for improved retrieval products, and the increased collaboration between the NLP and $\mathbb{R}$ communities will result in new techniques that will finally achieve the breakthrough in performance that is TIPSTER's goal.

\section{REFERENCES}

[1] Harman D. (Ed.).The Second Text REtrieval Conference (TREC-2). National Institute of Standards and Technology Special Publication 500-215, in press. 


\section{Best Automatic Adhoc}
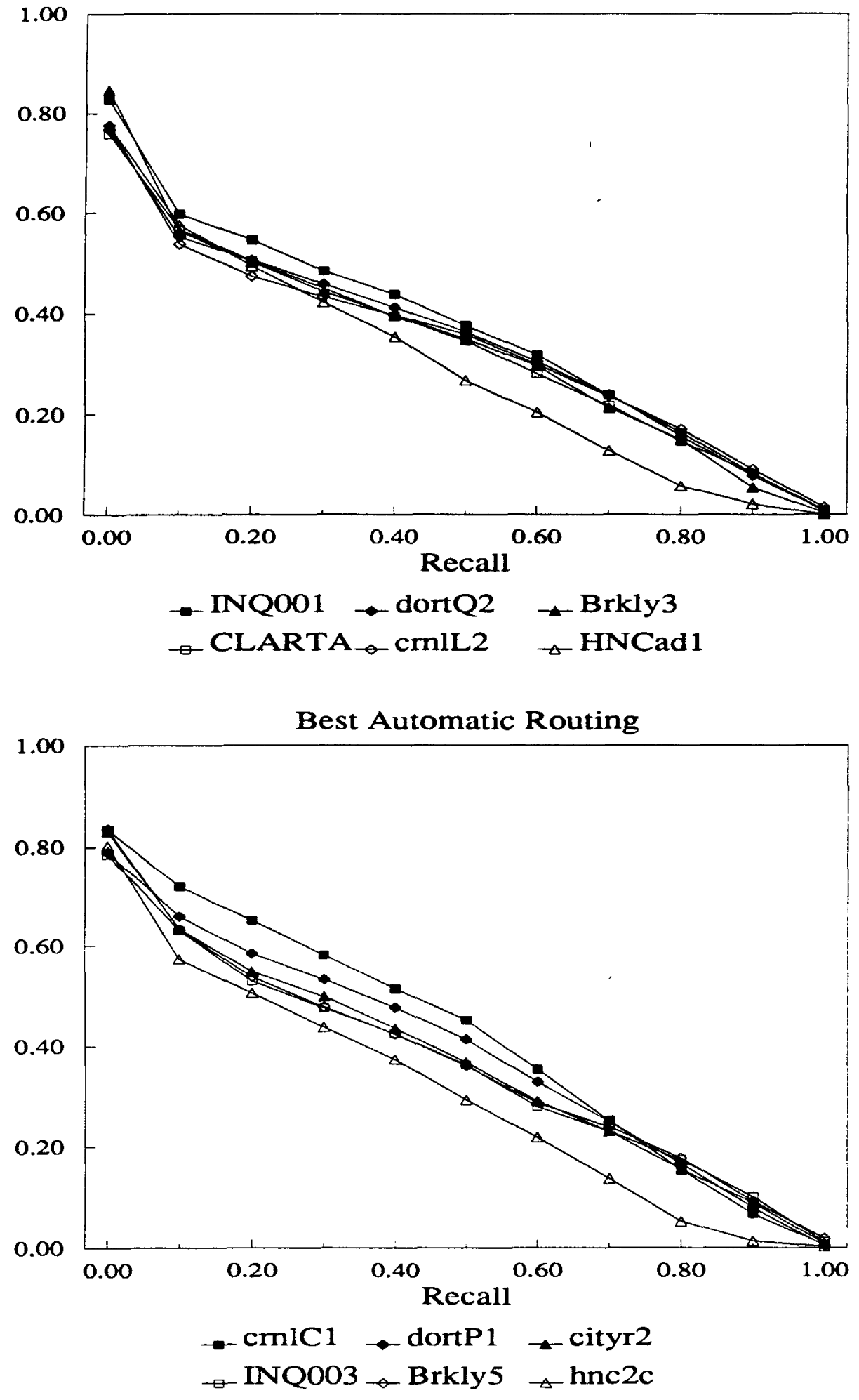

Figures 13 and 14: Best TREC-2 adhoc and routing performance using full collection 


\begin{tabular}{|c|c|c|c|c|c|c|c|c|}
\hline Topic & \multicolumn{8}{|c|}{ Top 8 Systems } \\
\hline 101 & rutcombl & VTcms2 & cmlV2 & INQ002 & dortQ2 & pircs3 & Brkly3 & $\overline{\text { CLARTM }}$ \\
\hline 102 & crnlL2 & crnlv2 & VTcms2 & siems3 & dortL2 & INQ002 & siems2 & CLARTM \\
\hline 103 & siems3 & siems2 & schaul & citril & crnlV2 & lsiasm & HNCad2 & HNCad 1 \\
\hline 104 & dortQ2 & CLARTM & CLARTA & pircs4 & pircs3 & dortL2 & HNCad2 & Isiasm \\
\hline 105 & citri2 & Isiasm & citri1 & siems2 & siems3 & crnlv2 & schaul & crnlL2 \\
\hline 106 & VTcms2 & INQ002 & INQ001 & TOPIC2 & pircs4 & pircs3 & CLARTM & dortL2 \\
\hline 107 & CnQst1 & $\mathrm{CnQst} 2$ & rutcomb1 & TOPIC2 & VTcms2 & INQ002 & rutfmed & CLARTM \\
\hline 108 & citril & dortQ2 & siems3 & VTcms2 & siems2 & HNCad2 & schaul & dortL2 \\
\hline 109 & dortL2 & crnlL2 & dortQ2 & CLARTA & CLARTM & pircs3 & cmlv2 & pircs4 \\
\hline 110 & INQ002 & INQ001 & Brkly3 & dortQ2 & nyuir3 & nyuir2 & cityau & siems2 \\
\hline 111 & CLARTA & CLARTM & INQ001 & dontQ2 & Brkly3 & siems2 & siems3 & pircs4 \\
\hline 112 & INQ002 & INQ001 & VTcms2 & nyuir2 & nyuir3 & HNCadl & HNCad2 & CnQst2 \\
\hline 113 & VTcms2 & cmlL2 & dortL2 & crniv2 & nyuir1 & siems2 & CLARTM & INQ002 \\
\hline 114 & INQ002 & cityau & VTcms2 & INQ001 & siems3 & siems2 & lsial & TOPIC2 \\
\hline 115 & nyuir2 & nyuir3 & nyuir1 & siems2 & dortL2 & cmlV2 & siems 3 & cmlL2 \\
\hline 116 & VTcms2 & CLARTA & HNCad2 & HNCad 1 & siems3 & siems2 & CLARTM & Brkly3 \\
\hline 117 & citri2 & citril & dortQ2 & INQ001 & TMC8 & lsiasm & gecrd2 & schaul \\
\hline 118 & nyuir2 & nyuir3 & nyuir1 & TOPIC2 & citymf & dontQ2 & CLARTA & INQ001 \\
\hline 119 & nyuir1 & nyuir 2 & nyuir3 & INQ002 & INQ001 & dortQ2 & citymf & VTcms2 \\
\hline 120 & citymf & nyuir2 & nyuir3 & nyuirl & CnQst2 & CnQst1 & VTcms2 & erima2 \\
\hline 121 & TOPIC2 & CLARTM & VTcms2 & Brkly3 & nyuir1 & prceol & INQ002 & rutfmed \\
\hline 122 & siems2 & siems 3 & INQ002 & INQ001 & dortQ2 & Brkly3 & CLARTM & cmlv2 \\
\hline 123 & nyuir1 & nyuir 2 & nyuir3 & CLARTA & INQ001 & INQ002 & CLARTM & pircs4 \\
\hline 124 & nyuir2 & nyuir 3 & nyuir1 & dortL2 & dortQ2 & INQ001 & Brkly3 & TMC9 \\
\hline 125 & cmlV2 & Brkly3 & cmll 2 & CLARTM & siems 3 & CLARTA & pircs4 & pircs 3 \\
\hline 126 & siems 3 & crnlL2 & siems2 & Brkly3 & cmlv2 & INQ002 & CLARTM & INQ001 \\
\hline 127 & cityau & Brkly3 & CLARTA & HNCad2 & INQ001 & INQ002 & siems2 & siems3 \\
\hline 128 & VTcms2 & CLARTA & siems3 & sicms2 & CLARTM & TOPIC2 & citril & Isiasm \\
\hline 129 & INQ001 & INQ002 & cityau & CLARTM & siems2 & Brkly3 & crnlL2 & CLARTA \\
\hline 130 & INQ002 & INQ001 & dortQ2 & crnlL2 & pircs 4 & CLARTM & dorL2 & pircs3 \\
\hline 131 & TOPIC2 & VTcms2 & HNCad1 & HNCad2 & siems 3 & Brkly3 & siems2 & INQ002 \\
\hline 132 & dortL2 & INQ001 & INQ002 & citril & citri2 & dortQ2 & HNCad2 & cmlL2 \\
\hline 133 & CnQst2 & CnQstl & rutcombl & pircs4 & INQ002 & pircs3 & cityau & INQ001 \\
\hline 134 & crnlL2 & dortL2 & nyuirl & nyuir2 & nyuir 3 & INQ002 & INQ001 & dortQ2 \\
\hline 135 & nyuir2 & nyuir3 & nyuir1 & Brkly3 & INQ001 & INQ002 & siems3 & siems2 \\
\hline 136 & VTcms2 & CnQstl & CnQst2 & CLARTM & pircs4 & CLARTA & dortQ2 & TOPIC2 \\
\hline 137 & CLARTA & nyuir2 & nyuir3 & Brkly3 & siems2 & siems3 & CLARTM & nyuir1 \\
\hline 138 & nyuir2 & nyuir3 & rutfmed & rutcomb1 & nyuir1 & schaul & gecrd2 & citril \\
\hline 139 & nyuir2 & nyuir3 & nyuir1 & VTcms2 & dortL2 & HNCad2 & dortQ2 & HNCadl \\
\hline 140 & nyuir2 & nyuir3 & nyuir1 & dortQ2 & dortL2 & INQ002 & siems 3 & siems2 \\
\hline 141 & VTcms2 & INQ002 & CnQst2 & INQ001 & Brkly3 & dortL2 & dortQ2 & CnQst1 \\
\hline 142 & dortQ2 & siems2 & cmlL2 & VTcms2 & siems3 & CLARTM & crnlv2 & Brkly3 \\
\hline 143 & INQ002 & INQ001 & siems2 & siems 3 & crnlL2 & crnlv2 & nyuir2 & nyuir3 \\
\hline 144 & VTcms2 & Brkly3 & citymf & crnlv2 & siems 3 & Isiasm & siems2 & HNCad2 \\
\hline 145 & cmlL2 & crnlV2 & dorLL2 & CLARTM & nyuir1 & siems3 & siems2 & dortQ2 \\
\hline 146 & Brkly3 & siems 3 & siems2 & Isiasm & cmlv2 & schaul & CLARTM & citril \\
\hline 147 & HNCad2 & HNCad1 & VTcms2 & citril & INQ002 & INQ001 & citymf & CLARTA \\
\hline 148 & Isiasm & cmlL2 & crnlv2 & siems2 & siems3 & Brkly3 & dorLL2 & dortQ2 \\
\hline 149 & nyuir1 & $\mathrm{CnQst2}$ & TOPIC2 & CnQstl & CLARTA & rutfmed & Brkly3 & rutcombl \\
\hline 150 & crnlL2 & dortQ2 & CLARTM & siems3 & INQ002 & INQ001 & crniv2 & siems2 \\
\hline
\end{tabular}

Table 1: The TREC-2 system rankings (using average precision) on individual topics 


\begin{tabular}{|l|c|c|}
\hline Relevant & & \\
Documents & HNCrt1 & INQ023 \\
\hline AP900115-0233 & - & - \\
AP900410-0212 & - & - \\
AP900905-0174 & - & - \\
SJMN91-06059027 & - & - \\
SJMN91-06072107 & - & - \\
AP900910-0280 & 1 & 360 \\
AP900914-0252 & 3 & 192 \\
AP901018-0234 & 4 & 288 \\
AP900822-0232 & 8 & 499 \\
SJMN91-06034021 & 10 & 23 \\
SJMN91-06063161 & 54 & 140 \\
AP900818-0028 & 208 & 549 \\
AP900924-0260 & - & 245 \\
AP900906-0203 & - & 322 \\
AP900903-0137 & 559 & 555 \\
AP900816-0111 & 849 & - \\
AP900829-0239 & 904 & 668 \\
\hline
\end{tabular}

Table 2: Ranks of retrieved relevant documents for topic 89

\begin{tabular}{|c|c|c|}
\hline $\begin{array}{c}\text { Relevant } \\
\text { Documents }\end{array}$ & Rank & Run Tag \\
\hline AP880214-0002 & 80 & INQ010 \\
AP880223-0008 & 26 & INQ013 \\
AP880622-0070 & 59 & INQ010 \\
AP880815-0056 & 40 & INQ010 \\
AP890522-0036 & 5 & INQ010 \\
AP891004-0223 & 39 & INQ013 \\
AP891130-0147 & 49 & INQ010 \\
AP891206-0043 & 23 & INQ013 \\
WSJ870325-0156 & 51 & INQ013 \\
WSJ900801-0135 & 124 & INQ011,INQ012 \\
ZF08-270-494 & 105 & HNCad2 \\
ZF08-305-768 & 3 & HNCad1 (all) \\
ZF08-386-296 & 57 & HNCad2 \\
\hline
\end{tabular}

Table 3: Relevant Documents for topic 121 (55 total relevant, 13 found TIPSTER 24-month) 